\title{
N93-26934
}

\section{Flow Instability in Particle-Bed Nuclear Reactors}

J. L. Kerrebrock*

\author{
Massachusetts Institute of Technology \\ PRESENTED AT NUCLEAR PROPULSION INTERCHANGE MEETING \\ NASA LEWIS RESEARCH CENTER, OCTOBER 22, 1992 \\ Abstract
}

The particle-bed core offers mitigation of some of the problems of solid-core nuclear rocket reactors. Dividing the fuel elements into small spherical particles contained in a cylindrical bed through which the propellant flows radially, may reduce the thermal stress in the fuel elements, allowing higher propellant temperatures to be reached. The high temperature regions of the reactor are confined to the interior of cylindrical fuel assemblies, so most of the reactor can be relatively cool. This enables the use of structural and moderating materials which reduce the minimum critical size and mass of the reactor. One of the unresolved questions about this concept is whether the flow through the particle-bed will be well behaved, or will be subject to destructive flow instabilities. Most of the recent analyses of the stability of the particle-bed reactor have been extensions of the approach of Bussard and Delauer, where the bed is essentially treated as an array of parallel passages, so that the mass flow is continuous from inlet to outlet through any one passage. A more general three dimensional model of the bed is adopted here, in which the fluid has mobility in three dimensions. Comparison of results of the earlier approach to the present one shows that the former does not accurately represent the stability at low Re. The more complete model presented here should be capable of meeting this deficiency while accurately representing the effects of the cold and hot frits, and of heat conduction and radiation in the particle-bed. II can be extended to apply to the cylindrical geometry of particle-bed reactors without difficulty. From the exemplary calculations which have been carried out, it can be concluded that a particle bed without a cold frit would be subject to instability if operated at the high temperatures desired for nuclear rockets, and at power densities below about 4 megawatts per liter. Since the desired power density is about $\mathbf{4 0}$ megawatts per liter, it can be concluded that operation at design exit temperature but at reduced power could be hazardous for such a reactor. But the calculations also show that an appropriate cold frit could very likely cure the instability. More definite conclusions must await calculations for specific designs.

* R.C. Maclaurin Professor of Aeronautics and Astronautics 


\section{Conclusions}

Comparison of three quite different approaches to modeling the stability of the particle-bed reactor, all with consistent physical assumptions, shows that a complete linear stability such as that presented here is in fact necessary for reliable prediction of the stability of the particle-bed reactor. The approach, termed here the Parallel-Stream model, where the reactor is assumed to be composed of a series of channels coupled only at their inlets and outlets, does not accurately represent the stability at low Re, nor does it represent the effect of heat conduction in the bed.

The model termed here (perhaps somewhat naively) the Complete Model should be capable of accurately representing the effects of the cold and hot frits, and of heat conduction and radiation in the particle bed. It can be extended to apply to the cylindrical geometry of particle-bed reactors without difficulty.

From the exemplary calculations which have been carried out, it can be concluded that a particle bed without a cold frit would be subject to instability if operated at the high temperatures desired for nuclear rockets, and at power densities below about 4 megawatts per liter. Since the desired power density is about $\mathbf{4 0}$ megawatts per liter, it can be concluded that opcration at design exit temperature but at reduced power could be hazardous for such a reactor. But the calculations also show that an appropriate cold frit could very likely cure the instability. More definite conclusions must await calculations for specific designs.

\section{Acknowledgments}

This research was supported by the Space Nuclear Propulsion Office of NASA Lewis Research Center.

The author wishes to acknowledge helpful discussions with Dr. John Clark of NASA Lewis, with Prof. Frank E. Marble, and with Mssrs. James Kalamas, David Suzuki, Timothy Lawrence and Jonathan Witter.

\section{References}

1) R. W. Bussard and R.D. DeLauer, Nuclcar Rocket Propulsion MoGraw-Hill, 1958 pp. 129133.

2) R. W. Bussard and R.D. DeLauer, Fundamentals of Nuclear Flight, McGraw-Hill, 1965 pp. 137-149.

3) E. Reshotko, AlAA Journal, vol 5, pp.1606-1615.

4) Bankston, Journal of Heat transfer, Nov 1970 pp. 569-579. 
5) M. Charmchi, J.W. McKelliget, M. Rand, G.Maise, "Thermo-Hydraulic Characteristics of Gas-Cooled Particle Bed Reactors" Proc. of Fourth International Topical Meeting on Nuclear reactor Thermal-Hydraulics(NURETH-IV), Karlsruhe, FRG, Vol 1, 1989, pp 139-145.

6) J. K.Witter, D.D. Lanning, J.E. Meyer, "Flow Stability Analysis of a Particle-Bed Reactor Fuel Element", personal communication October 1992.

7) S. Ergun, "Fluid Flow Through Packed Columns", Chemical Engineering Progress, vol 48 no 2 pp. 89-94, February 1952

8) Y. B. Zel'dovich, Y.P. Raizcr, Physics of shock Waves and High-Temperature

Hydrodynamic Phenomena, Academic Press 1966. 
SCHEMATIC OF PARTICLE-BED

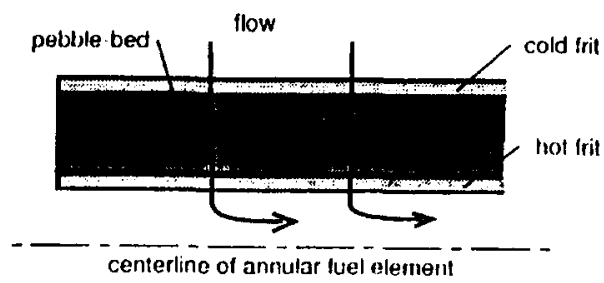

\section{GOVERNING EQUATIONS}

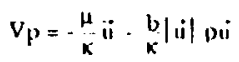

$$
\begin{aligned}
& \mu=1.50 \frac{(1-\varepsilon)^{2} \mu\left(T_{p}\right)}{\varepsilon^{3} D_{p}^{2}} \\
& b=1.75 \stackrel{(1 c)}{f^{3}} \quad 1
\end{aligned}
$$

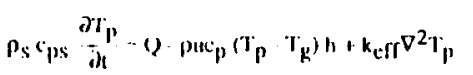

$$
\begin{aligned}
& k_{\text {eff }}=k_{\text {comol }}+k_{\text {rand }}-k_{\text {cond }}+1_{3} \sigma \mathrm{D}_{p} \mathrm{p}_{\mathrm{p}}{ }^{3}
\end{aligned}
$$

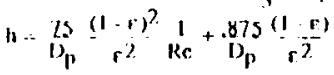

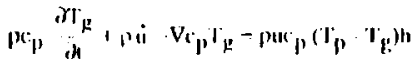

$$
\begin{aligned}
& d \mu+v(p u)=0 \\
& \text { l' ikl }
\end{aligned}
$$


NON-DIMENSIONAL GOVERNING EQUATIONS

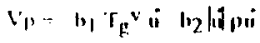

$$
\begin{aligned}
& \text { (III) } \\
& h_{1}=\frac{\mu_{n}(0) u_{0}(0) !}{S_{0}(0)}
\end{aligned}
$$

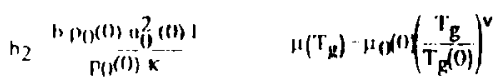

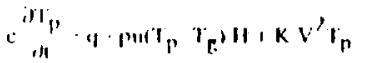

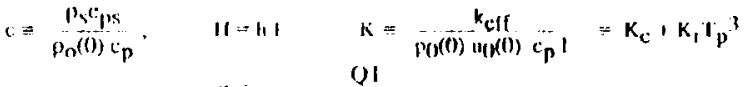

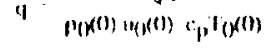

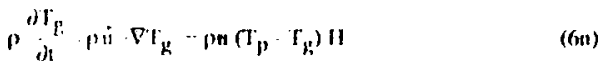

$$
\begin{aligned}
& \text { Si }, 0 \text {, } \\
& p \cdot \varphi_{k}
\end{aligned}
$$

\section{ZEROTH-ORDER OR STEADY SOLUTION}

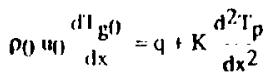

$$
\begin{aligned}
& T_{g(1)}=1+q x \\
& r_{p w}-1+q x+\frac{q}{H}
\end{aligned}
$$

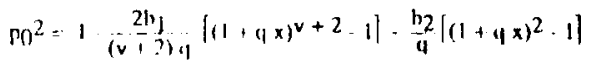

$$
\begin{aligned}
& \text { (i) }-p_{g^{\prime}} \\
& \text { 11(i) }-\frac{1}{p_{0}}
\end{aligned}
$$




\section{FIRST ORDER}

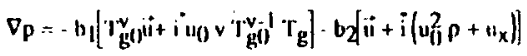

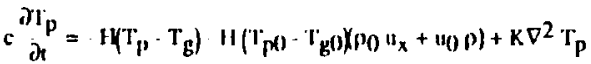

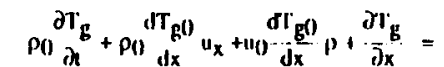

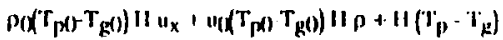

$\left.\frac{\partial \rho}{\partial t}+\rho \theta \nabla \cdot i+\frac{d \rho \theta}{d x} \|_{x}+w 0\right) \frac{\partial p}{\partial x}+\frac{d u(p)}{d x} \rho=0$

$$
p=P O T_{E} \cdot T_{g O n}
$$

(18)

Variables are: $p, p, T_{g}, i$ and $T_{p}$
}

\section{PARAMETERS}

\section{Dimensionless Parameters :}

from 1n, $v, b_{1}, b_{2}$

from $3 n, \quad q, c, H, K_{c}, K_{r}$

Operating Parameters :

$T_{90}$ (exit) $=3000 \mathrm{~K}$

$p_{0}$ (exit) $=100 \mathrm{~atm}$

$Q=4 \times 10^{10}$ wat/m3

Design Parameters :

1 $=0.01 \mathrm{~m}$

$D_{p}=.5 \times 10^{3} \mathrm{~m}$

Stability Parameters :

$$
R_{e}=\frac{P_{0}(0) \mu_{0}(0) D_{p}}{\mu(0)}=\frac{D_{p} Q I}{c_{p} T_{0} \mu(0) \times}
$$


APPROACHES TO INSTABILITY ANALYSIS
1) Parallel Flow Instability
2) Local Instability Analysis
3) Full Stability Analysis

\section{PARALLEL-STREAM INSTABILITY}

Instability is possible if po(1) increases with mass flow density for fixed $Q$ and $p_{0}(0)$. Hence:

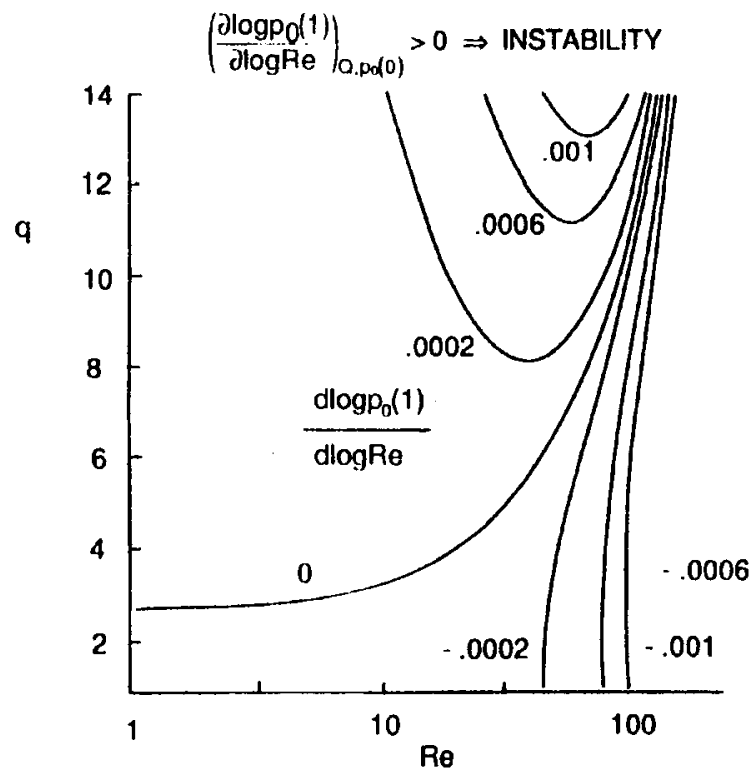




\section{COMPLETE INSTABII.ITY MODEL}

$$
\begin{aligned}
& p(x, y, z, 1)=p(x)=0 \mid k, r)+\infty 1 \\
& \frac{d p}{d x}=\left(b_{1} T_{g}^{v}(1)+2 b_{2}\right) n_{x}-\left(b_{1}+n_{0} \vee T_{g}^{v}(1) T_{g} \cdot\left(b_{2} w_{0}^{2}\right) \rho\right. \\
& \frac{d T_{g}}{d x}=H\left(T_{p}-T_{g}\right) \cdot\left(\rho(\omega) T_{g}\right.
\end{aligned}
$$

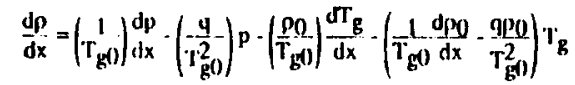

$$
\begin{aligned}
& \frac{d u_{x}}{d x}=-\left(\frac{1 d \rho_{0}}{\rho(0) d x}\right) u_{x} \cdot\left(\frac{w_{0}}{\rho 0}\right) \frac{d \rho}{d x} \cdot\left(\frac{1}{\rho_{0}} \frac{d u_{0}}{d x}+\underset{\rho_{0}}{\omega}\right) \rho\left(\begin{array}{c}
k_{f}^{2} \\
m_{2}+b_{1} T_{g(0)}^{Y}
\end{array}\right) p \\
& K \frac{d y}{d x}=(k k f+c(1)+11) T_{p} \cdot H T_{g}+(4 p(0)) u_{x}+\left(4\left(u_{0}\right)\right) \rho \\
& \frac{d r_{p}}{d x}=y
\end{aligned}
$$

Approximate Case Neglecting Conduction in $\mathrm{x}$ :

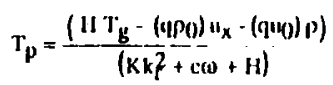
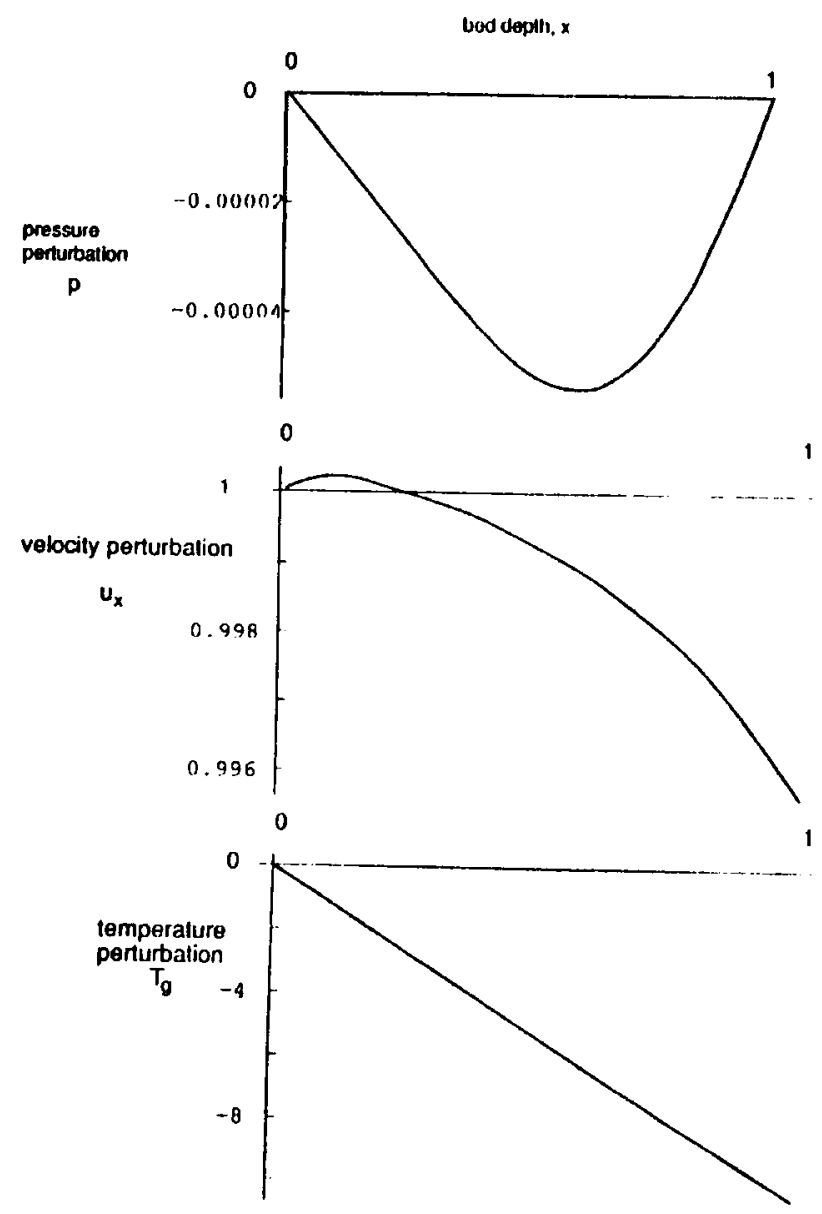


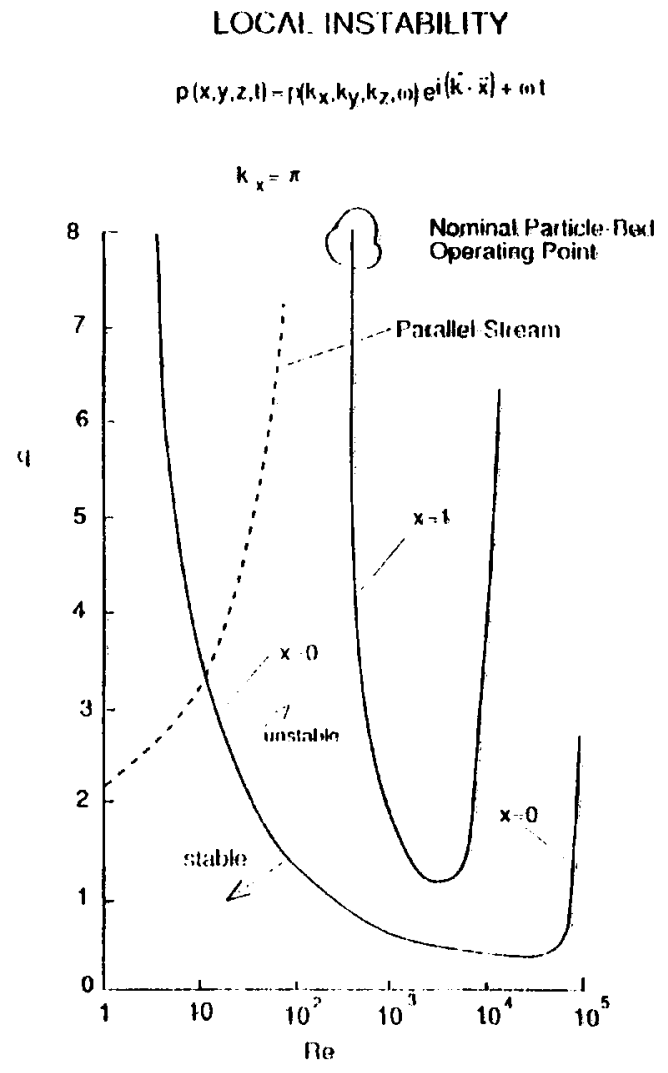

COMPLETI INSIABILITY MODEL

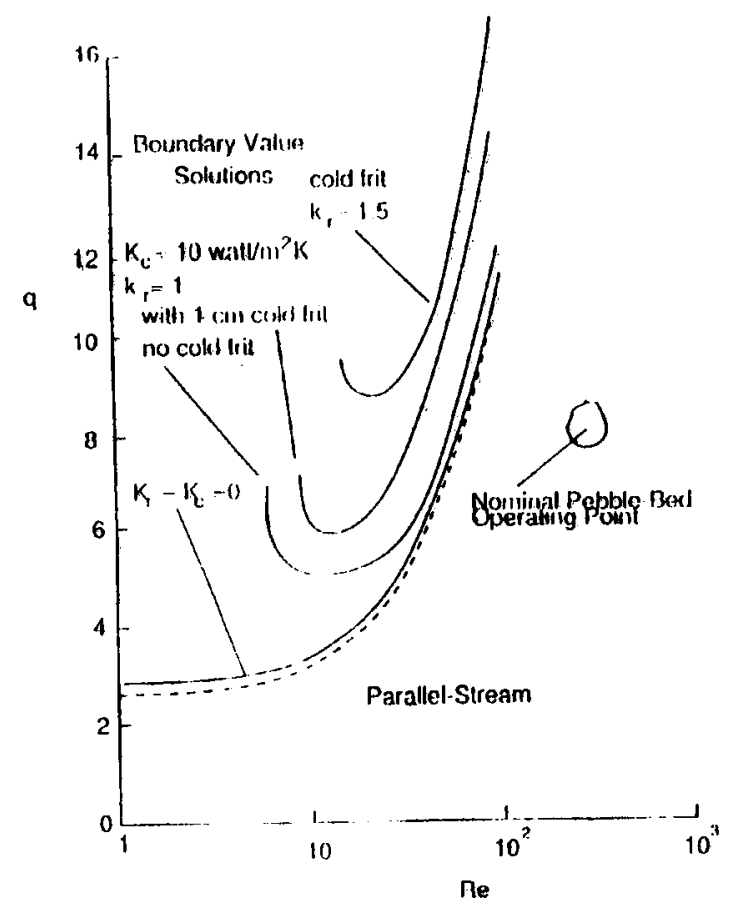

